\title{
1 Impact of Premorbid Infection on Onset and Disease Activity of Rheumatoid Arthritis
}

3 Ruijun Zhang ${ }^{1,2 \#}$, Jing $\mathrm{Li}^{1,2 \#}$, Jiali Chen ${ }^{1,2}$, Xiaomei Chen ${ }^{1,3}$, Xue $\mathrm{Li}^{1,2}$, Chun ${ }^{1,2}{ }^{1,2}$ Yuan $\mathrm{Jia}^{1,2}$, 4 Yunshan $\mathrm{Zhou}^{1,2}$,Limin Ren ${ }^{1,2}$, Lijun $\mathrm{Wu}^{1,3}, \mathrm{Jing} \mathrm{He}^{1,2^{*}}$,Zhanguo Li ${ }^{1,2^{*}}$

5 1. Department of Rheumatology \& Immunology, Peking University People's Hospital, Beijing 6 100044, China.

7 2. Beijing Key Laboratory for Rheumatism Mechanism and Immune Diagnosis (BZ0135), Beijing, 8 China.

9 3. Department of Rheumatology\& Immunology, People's Hospital of Xinjiang Uygur Autonomous 10 Region, Wulumuqi 830000, China.

11 *Corresponding authors: Department of Rheumatology and Immunology, Peking University People's

12 Hospital, 11 Xizhimen St. Beijing 100044, China. Email: Pro. Jing He: hejing1105@126.com; Pro.

13 Zhanguo Li: zgli99@aliyun.com

14 "Ruijun Zhang and Jing $\mathrm{Li}$ equally contributed to this work. Email: Zhang ruijun: 15 zhangruijunyc@126.com; Li jing: muzijing1026@163.com 


\section{Abstract}

21 Objective. Infections have been implicated in rheumatoid arthritis (RA) development. However, the

22 impact of premorbid infection on initiation and perpetuation of RA has not been well elucidated.

23 Thus, we sought to conduct a large scale on-site survey to study whether premorbid infection may

24 trigger RA and influence status of the disease.

25 Methods. Premorbid infectious events were collected in cohort of 902 RA patients from December

262015 to June 2016. Type of infections prior to RA onset and its possible effects on disease status were analyzed.

28 Result. Three hundred and thirty-four out of 902 patients (37.03\%) experienced infections within one

29 month preceding RA onset. The most frequent infections were respiratory (16.08\%), intestinal

$30(11.09 \%)$ and urinary tract $(9.87 \%)$ infection, respectively. The infection was associated with

31 increased disease activity. Early onset was found in patients with urinary infection. High disease

32 activity risk was increased in patients who pre-exposure to urinary infection (OR=3.813,

$3395 \% \mathrm{CI}=1.717-12.418)$ and upper respiratory infection $(\mathrm{OR}=2.475,95 \% \mathrm{CI}=0.971-6.312)$.

34 Conclusion. Pre-exposure infections are associated with development of RA. Severe disease status

35 of RA and persistent of active disease status are related to preceding infections.

36 Keywords: Premorbid infection, Rheumatoid Arthritis, RA onset, disease activity 


\section{Introduction}

46 Rheumatoid arthritis (RA) is a common autoimmune disease characterized by joint destruction and

47 auto-antibodies production.[1] Many studies have demonstrated that infectious agents may contribute

48 to the initiation or perpetuation of RA through a variety of mechanisms. Infection can cause a local

49 inflammatory response. The innate immune system could also be affected by infections agents and

50 then cause RA onset, for instance, pathogen-associated molecular pattern receptors, especially the

51 Toll-like receptors (TLRs) could release inflammatory mediators rapidly after recognizing some

52 preserved structures in bacteria and other infectious agents [2].

53 Although a definite causative link between a specific infectious agent and the disease has not

54 been established, several arguments support such a possibility. First, in the absence of a certain

55 pathogen, the spectrum of microorganisms involved in triggering RA may include poly-microbial

56 communities or the cumulative effect of bacterial or virus factors [3]. Secondly, infections didn't lead

57 to RA in all cases, but initiate it in a certain subset of patients who was born with a genetic

58 susceptibility [4-7]. Thirdly, some arthritis occurred based on pre-exposure to microorganism.

59 Several animal models of arthritis are dependent on TLR2, TLR3, TLR4 or TLR9, for instance,

60 rodents injected with streptococcal cell walls (TLR2 ligand) develop severe polyarticular arthritis

61 and TLR4 ligand also play a role in passive $\mathrm{K} / \mathrm{BxN}$ arthritis [8]. Many studies have shown that

62 components derived from infectious agents can cause autoimmune reaction by molecular mimicry

63 and other mechanisms. Epstein-Barr virus (EBV) is a polyclonal B lymphocyte activator which can

64 increase the production of RF [4]. Oral pathogens may trigger the production of disease-specific

65 autoantibodies and arthritis in susceptible individuals. It has been shown recently that RA is 
associated with exposure to some microorganism such as Aggregatibacter actinomyce-temcomitans

(Aa) [1].

In this study, we sought to conduct a large-scale survey to explore potential infectious agents

which might initiate RA and the clinical consequence of this disease.

\section{METHODS}

72 Patients Survey results were collected from 902 RA patients admitted to the Department of

73 Rheumatology and Immunology, People's Hospital, Peking University, between December 2015 and

74 June 2016. All the studied patients fulfilled the American College of Rheumatology/European

75 League Against Rheumatism Classification criteria for RA in 2010, and written informed consent

76 was obtained.

The clinical data were recorded including tender and swollen 28-joint counts, general health on

78 visual analog scales, erythrocyte sedimentation rate (ESR), Health Assessment Questionnaire

79 (HAQ), 28-joint Disease Activity Score (DAS28) and the infectious agents one month before RA

80 onset.

81 The questionnaire including age, sex, disease duration, age at symptom, smoking status, DAS28

82 using the ESR at enrolment and treatments (one DMARD, more than one DMARDs, DMARDs plus

83 low-dose glucocorticoid and bDMARDs). Only premorbid infectious agents of the RA patients were

84 carefully recorded in this study.

85 Statistical analyses Analysis of covariance and multivariate logistic regression analysis were applied 
86 to compare the disease activity in patients with or without prior infections. T test or ANOVA was

87 used to analyze the data. The categorical variables were compared with chi-squared test. Multinomial

88 logistic analysis was used to find risk factor which perhaps affected the current disease activity in RA

89 patients. Data was expressed as mean \pm stand errors for continuous variables. The SPSS statistical

90 package, version 23.0 was used for all statistical analyze, and p value less than 0.05 was considered

91 statistically significant.

93 Results

\section{1. Prevalence of infections in RA}

95 Within one month prior to RA onset, 37.03\% (334/902) patients experienced infections, and the most 96 frequent sites were respiratory (16.08\%), intestinal (11.09\%) and urinary (9.87\%), respectively

97 (Table 1).

\section{2. Patients in severe disease status showed high prevalence of infections}

99 Four-hundred and ninety out of 902 RA patients with complete clinical data were analyzed in this 100 study. These patients were divided into two groups based on DAS28 (DAS28<3.2 as group 1; 101 DAS28 $\geq 3.2$ as group 2). Compared with patients in group 2, patients in group 1 showed high 102 prevalence of non-premorbid infection $\left(\mathrm{X}^{2}=18.193, \mathrm{P}=0.000\right)$ (Table 2). Notably, patients with high 103 disease activity suffered more pre-exposure of respiratory, intestinal and urinary infections $(\mathrm{P}=0.000$, $104 \mathrm{P}=0.000, \mathrm{P}=0.023$; respectively) (Table 2). Besides, higher ESR and CRP were observed in patients 105 with higher DASD28 scores (Table 3).

\section{3. Disease Activity was associated with premorbid infection.}


107 In our study, patients showed higher DAS28 in urinary $(\mathrm{P}=0.000)$ and respiratory $(\mathrm{P}=0.001)$ infection

108 groups (Table4) before adjusting confounding factors such as the different therapies, age and

109 smoking status which can affect disease activity.

110 One hundred and forty-five RA patients experienced respiratory tract infections one month prior

111 to onset of the disease. Among these patients, 13.30\% (120/902) patients showed upper respiratory

112 tract infection while $2.77 \%$ (25/902) patients with lower respiratory tract infection. The number of

113 tender and swollen joints (Fig 1A and B), HAQ scores (Fig 1D) and DAS 28 (Fig 1E) were higher in

114 patients who had the respiratory tract infection compared with patients who had no infection before

115 RA occurred. Furthermore, DAS28 was higher in respiratory infection group after adjusting for the

age $(\mathrm{P}=0.002)$ and smoking $(\mathrm{P}=0.002)($ Table4 $)$

117 There were 89 patients with urinary infection who developed RA in one month before disease

118 initiation. More deformed joints (Fig 1C) were found in patients who had premorbid urinary

119 infection. The age at onset was younger in patients who had urinary infection. (Fig 1F) DAS28 was

120 still higher in urinary infection group after adjusting for the therapy type $(\mathrm{P}=0.000)$ and smoking

$121(\mathrm{P}=0.002)$ group $($ Table4).

122 Intestinal infection occurred in 100 patients who developed RA. No difference was observed in

123 these patients compared to patients with no infection. (Fig 1A-F) After adjusting age and smoking,

124 DAS28 didn't show significant difference between intestinal infection group and no infection group

125 (Table4).

126 4. Potential risk factors for high disease activity.

127 The multinomial logistic regression was trained for predicting the disease activity with the factors 
128 which showed statistical significance in single-factor analysis (Supplementary Table 2). These model

129 parameters were for the low, moderate and high levels of disease activity, measured relative to the

130 remission level (reference outcome). High disease activity risk was increased in patients who had

131 urinary infection $(\mathrm{OR}=3.813,95 \% \mathrm{CI}=1.717-12.418)$ (Figure 2), and upper respiratory infection

$132(\mathrm{OR}=2.475,95 \% \mathrm{CI}=0.971-6.312) \quad($ Figure 2$)$.

\section{Discussion}

135 There is increasing awareness that mucosal surfaces, including the gut and lungs, was sites of disease

136 initiation in RA [8]. Recent studies showed that infectious agents including virus and bacteria

137 infection had been associated with several kinds of autoimmune disease [7,10-12]. For instance,

138 upper respiratory tract and other infections are well-known risk factors for multiple sclerosis [13].

139 However, it was not clearly whether infectious agents play the causative role in the onset or outcome

140 of autoimmune disease, this is mainly due to the lack of strictly perspective epidemiological study.

141 And even in animal models, these relationships are complex and depend on the timing of exposure,

142 antigen type and genetic background [14]. In our study, the age of disease onset was younger in

143 patients who had urinary tract infection, which perhaps indicates that RA occurred earlier in patients

144 with this pre-exposure infection and later in the other patients.

145 It has been certified that many virus can play a role in the production of auto-antibodies such as

146 anti-cyclic citrullinated peptide [15]. Infections are known to cause or enhance autoimmunity

147 through expansion of auto-reactive T-cell clones by molecular mimicry and enhanced antigen

148 presentation [14]. The patients with infection events during the disease duration could have advanced 
149 RA status [16]. To our knowledge, there was no study to prove the relationship between the 150 premorbid infection history and onset or outcomes of RA in large populations. Here, we made the 151 first report that analyzed this relationship in RA patients from outpatient of department of 152 rheumatology and immunology in People's Hospital, Peking University.

153 There were many factors reflected the disease activity in RA, such as the number of tender or 154 swollen joints, ESR, CRP and so on. Patients with respiratory tract infection had higher DAS28 and 155 more swollen/tender joints. This probably because of respiratory tract infection was mainly caused 156 by viruses. Acute viral infection in adults have long been suggested to induce transient autoimmune 157 responses, including generation of autoantibody [7]. As reported in a recent study, Arleevskaya et al 158 found that higher percentages of first-degree healthy relatives (HR) than health control (HC) had 159 upper respiratory and urinary tract infections. During10-year follow-up, 26 out of 251 (10.36\%) HR 160 subjects developed to RA, while no RA was found in HC group [4]. In our study, we found that $1619.87 \%$ (89/902) patients had pre-exposure of urinary tract infection and $13.30 \%$ (120/902) patients 162 with upper respiratory infection. Besides, the patients with urinary infection were more likely to stay 163 in disease activity stage and have more deformity joints. Moreover, the patients with respiratory 164 infection had higher disease activity compared with no infection patients.

165 In fact, it is impossible to make a causal link between a specific pathogen and the disease. Our 166 study has several limitations. First, because the study was done in a retrospective manner, the 167 patients who had no complete clinical data were excluded from this study. Second, the number of the 168 studied patients was not large enough to see the statistical difference in clinical features and odds 169 ratio in lower respiratory tract infection subgroup patients. It may be due to this study group with 
very few patients. Third, our studied patients may have selection bias because it was performed in a single university hospital. In order to determine the impact of premorbid infectious agents for RA outcome, the disease activity at RA onset and radiographic joint damage should be followed up in a larger prospective study.

Acknowledgements: No.

Conflict of interest statement: The authors declare no conflicts of interest.

Funding: This work was supported by National Natural Science Foundation of China. [NSFC

\section{【Reference】}

[1] Konig MF, Abusleme L, Reinholdt J, Palmer RJ, Teles RP, Sampson K, et al. Aggregatibacter actinomycetemcomitans-induced hypercitrullination linksperiodontal infection to autoimmunity in rheumatoid arthritis. SicTransl Med, 2016; 8(369):369ra176.

[2] Gray S. Firestein, Ralph C. Budd, Sherine E. Gabriel, et al. Kelley's Textbook of Rheumatology. Singapore: Elsevier PteLtd, 2015:1139-1142.

[3] Arleevskaya MI, Kravtsova OA, Lemerle J, Renaudineau Y, Tsibulkin AP. How Rheumatoid Arthritis Can Result from Provocation of the Immune System by Microorganisms and Virus. Front Microbiol. 2016; 17(7):1296.

[4] Arleevskaya, MI, Gabdoulkhakova, AG, Filina, YV, Miftakhova RR, Bredberg A, Tsybulkin AP. A transient peak of infections during onset of rheumatoid arthritis: a 10-year prospective cohort study. BMJ Open. 2014, 4(8):e005254.

[5] Leirisalo-Repo, M. Early arthritis and infection. Curr Opin Rheumatol. 2005; 17: 433-439.

[6] Benedek TG. The history of bacteriologic concepts of rheumatic fever and rheumatoid arthritis. Semin Arthritis Rheum. 2006; 36(2):109-23.

[7] Ori Barzilai, Maya Ram, Yehuda Shoenfeld. Viral infection can induce the production of autoantibodies. Curr Opin Rheumatol. 2007; 19(6):636-43.

[8] Choe JY, Crain B, Wu SR, Corr M. Interleukin 1 receptor dependence of serum transferred arthritis can be circumvented by toll-like receptor 4 signaling. J Exp Med, 2003; 197:537. 
200 alters natural killer cell homeostasis and is shared in several autoimmune diseases. Cell Host 201 Microbe. 2016; 19(3):400-8.

202 [10] Brusca SB, Abramson SB, Scher JU. Microbiome and mucosal inflammation as extra-articular 203 triggers for rheumatoid arthritis and autoimmunity. Curr Opin Rheumatol. 2014; 26:101-107.

204 [11] Haleniusm A, Henge H. Human cytomegalovirus and autoimmune disease. Biomed Res Int. 205 2014:472978. doi: 10.1155/2014/472978. Epub 2014 Apr 29

206 [12] IgoeA, Scofield RH. Autoimmunity and infection in Sjögren's syndrome. Curr Opin 207 Rheumatol.2013; 25(4):480-7.

208 [13] Bulijevac D, Flach HZ, Hop WC, Hijdra D, Laman JD, Savelkoul HF, et al. Prospective study 209 on the relationship between infections and multiple sclerosis exacerbations. Brain. 2002; 125(pt 210 5):952-60.

211 [14] MünzC, Lünemann JD, Getts MT, Miller SD. Antiviral immune responses: triggers of or 212 triggered by autoimmunity? Nat Rev Immunol. 2009; 9(4): 246-258.

213 [15] Costenbader KH, Karlson EW. Epstein-Barr virus and rheumatoid arthritis: is there a link? 214 Arthritis Res Ther. 2006;8:204

215 [16] Iguchi-Hashimoto M, Hashimoto M, Fujii T, Hamaguchi M, Furu M, Ishikawa M, et al. The 216 association between serious infection and disease outcome in patients with rheumatoid arthritis. Clin 217 Rheumatol. 2016; 35(1):213-8. 
Table 1 The type of premorbid infections in RA patients

\begin{tabular}{lcc}
\hline Infection types & Cases & Percentage (\%) \\
\hline No infection & 568 & 62.97 \\
Respiratory & 145 & 16.08 \\
$\quad$ Upper & 120 & 13.30 \\
$\quad$ Lower & 25 & 2.77 \\
Intestinal & 100 & 11.09 \\
Urinary & 89 & 9.87 \\
\hline
\end{tabular}


Table 2 Prevalence of infection in RA patients with different disease activity

\begin{tabular}{ccccc}
\hline Infection types & $\begin{array}{c}\text { Group 1 }(\mathrm{n}=244) \\
\text { DAS28<3.2 } \\
(\mathrm{n}, \%)\end{array}$ & $\begin{array}{c}\text { Group 2 }(\mathrm{n}=246) \\
\text { DAS28 } \geq 3.2 \\
(\mathrm{n}, \%)\end{array}$ & $\mathrm{X}^{2}$ & P value \\
\hline No infection & $201(82.4)$ & $161(65.4)$ & 18.193 & 0.000 \\
Respiratory & $20(8.2)$ & $39(15.9)$ & 30.384 & 0.000 \\
Intestinal & $10(4.1)$ & $17(16.9)$ & 125.390 & 0.000 \\
Urinary & $13(5.3)$ & $29(11.8)$ & 7.518 & 0.023 \\
\hline
\end{tabular}


Table 3 Clinical characteristics and demographics of RA patients

\begin{tabular}{|c|c|c|c|c|c|}
\hline \multicolumn{2}{|c|}{ Characteristic } & $\begin{array}{c}\text { Group } 1 \\
(\mathrm{DAS} 28<3.2) \\
(\mathrm{n}=244)\end{array}$ & $\begin{array}{c}\text { Group } 2 \\
\text { (DAS28 } \geq 3.2) \\
(n=294)\end{array}$ & Statistic & $\mathrm{P}$ value \\
\hline \multicolumn{2}{|c|}{ Male $^{\mathrm{c}}, \mathrm{n}(\%)$} & $50(20.5)$ & $50(20.3)$ & 4.601 & 0.100 \\
\hline \multicolumn{2}{|c|}{$\operatorname{Age}^{\mathrm{a}}$ (years) } & $54 \pm 14$ & $55 \pm 13$ & -0.281 & 0.779 \\
\hline \multicolumn{2}{|c|}{ Disease duration $^{\mathrm{b}}$ (years) } & $3(2,5)$ & $8(3,22.5)$ & -0.953 & 0.340 \\
\hline \multicolumn{2}{|c|}{ Age at diagnosis ${ }^{\mathrm{b}}$ (years $)$} & $44 \pm 14$ & $45 \pm 15$ & -0.781 & 0.435 \\
\hline \multicolumn{2}{|c|}{$\mathrm{ESR}^{\mathrm{b}}(\mathrm{mm} / \mathrm{H})$} & $11(7,18)$ & $33(19,55)$ & -13.337 & 0.000 \\
\hline \multicolumn{2}{|c|}{$\mathrm{CRP}^{\mathrm{b}}(\mathrm{mg} / \mathrm{L})$} & $2.68(1.44,4.87)$ & $\begin{array}{c}9.58(3.31 \\
23.19)\end{array}$ & -10.531 & 0.000 \\
\hline \multicolumn{2}{|c|}{ Anti-CCP negative $^{c}, \mathrm{n}(\%)$} & $\begin{array}{c}37 \\
(37 / 223,16.6 \%)\end{array}$ & $\begin{array}{c}36 \\
(36 / 154,23.4 \%)\end{array}$ & 2.686 & 0.101 \\
\hline \multicolumn{2}{|c|}{ Anti-CCP antibody ${ }^{\mathrm{b}}$ ( U/L ) } & $\begin{array}{c}167.53 \\
(57.2, \quad 224.14)\end{array}$ & $\begin{array}{c}165 \\
(38.24, \\
225.21)\end{array}$ & -0.419 & 0.675 \\
\hline \multicolumn{2}{|c|}{$\mathrm{HAQ}^{\mathrm{b}}$} & $1(0,3)$ & $5(1,12)$ & -10.013 & 0.000 \\
\hline \multirow{3}{*}{ Smoking Status ${ }^{c}$} & Never smokers & $\begin{array}{c}142 \\
(142 / 209,67.9)\end{array}$ & $\begin{array}{c}157 \\
(157 / 215,73.0)\end{array}$ & \multirow{3}{*}{1.350} & \multirow{3}{*}{0.509} \\
\hline & Passive smokers & $\begin{array}{c}23 \\
(23 / 209,11.0)\end{array}$ & $\begin{array}{c}19 \\
(19 / 215,8.8)\end{array}$ & & \\
\hline & Active smokers & $\begin{array}{c}44 \\
(44 / 209,21.1)\end{array}$ & $\begin{array}{c}39 \\
(39 / 215,18.1)\end{array}$ & & \\
\hline \multirow{5}{*}{ Current Treatment $^{\mathrm{c}}$} & One DMARD & $\begin{array}{c}56 \\
(56 / 222,25.2)\end{array}$ & $\begin{array}{c}38 \\
(38 / 203,18.7)\end{array}$ & \multirow{5}{*}{15.904} & \multirow{5}{*}{0.001} \\
\hline & More than one & 138 & 109 & & \\
\hline & DMARD & $(138 / 222,62.2)$ & $(109 / 203,53.7)$ & & \\
\hline & $\begin{array}{l}\text { DMARDs + } \\
\text { glucocorticoid }\end{array}$ & $\begin{array}{c}14 \\
(14 / 222,6.3)\end{array}$ & $\begin{array}{c}23 \\
(23 / 203,11.3)\end{array}$ & & \\
\hline & bDMARDs & $\begin{array}{c}14 \\
(14 / 222,6.3)\end{array}$ & $\begin{array}{c}33 \\
(33 / 203,16.3)\end{array}$ & & \\
\hline
\end{tabular}

(ESR, erythrocyte sedimentation rate; CRP, C-reactive protein; anti-CCP, anti-citrullinated peptide antibodies; a, Data is described as mean $\pm \mathrm{SD}$, analysis with t-test; $b$, Data are reported as median with top and bottom quartile, nonparametric test is used for analysis; c, Chi-squared testis used.) 
Table 4 Differences of DAS28 between infectious groups and no infection group

\begin{tabular}{ccccc}
\hline & No-infection & Urinary & Respiratory & Intestinal \\
\hline $\begin{array}{c}\text { Before } \\
\text { adjusted }\end{array}$ & $3.25 \pm 0.07$ & $3.97 \pm 0.19^{\triangle}$ & $3.78 \pm 0.17^{\triangle}$ & $3.56 \pm 0.26$ \\
$\begin{array}{c}\text { Adjusting for confounding factors } \\
\text { Therapy }\end{array}$ & & & \\
Age $^{\text {a }}$ & $3.20 \pm 0.07$ & $3.91 \pm 0.20^{\Delta}$ & - & - \\
Smoking $^{\text {a }}$ & $3.26 \pm 1.41$ & - & $3.80 \pm 1.450^{*}$ & $3.58 \pm 1.50$ \\
\hline
\end{tabular}

Analysis of covariance was applied for adjusting confounding factors; a: Adjusted for therapy; $b$ : Adjusted for age; c: Adjusted for smoking; - : Cannot be adjusted because of having an interaction effect compared with no-infection; Analysis of covariance was used between no-infection group and other infectious groups, ${ }^{*}: \mathrm{P}<0.05^{\triangle}$ : $\mathrm{P}<0.001$. 

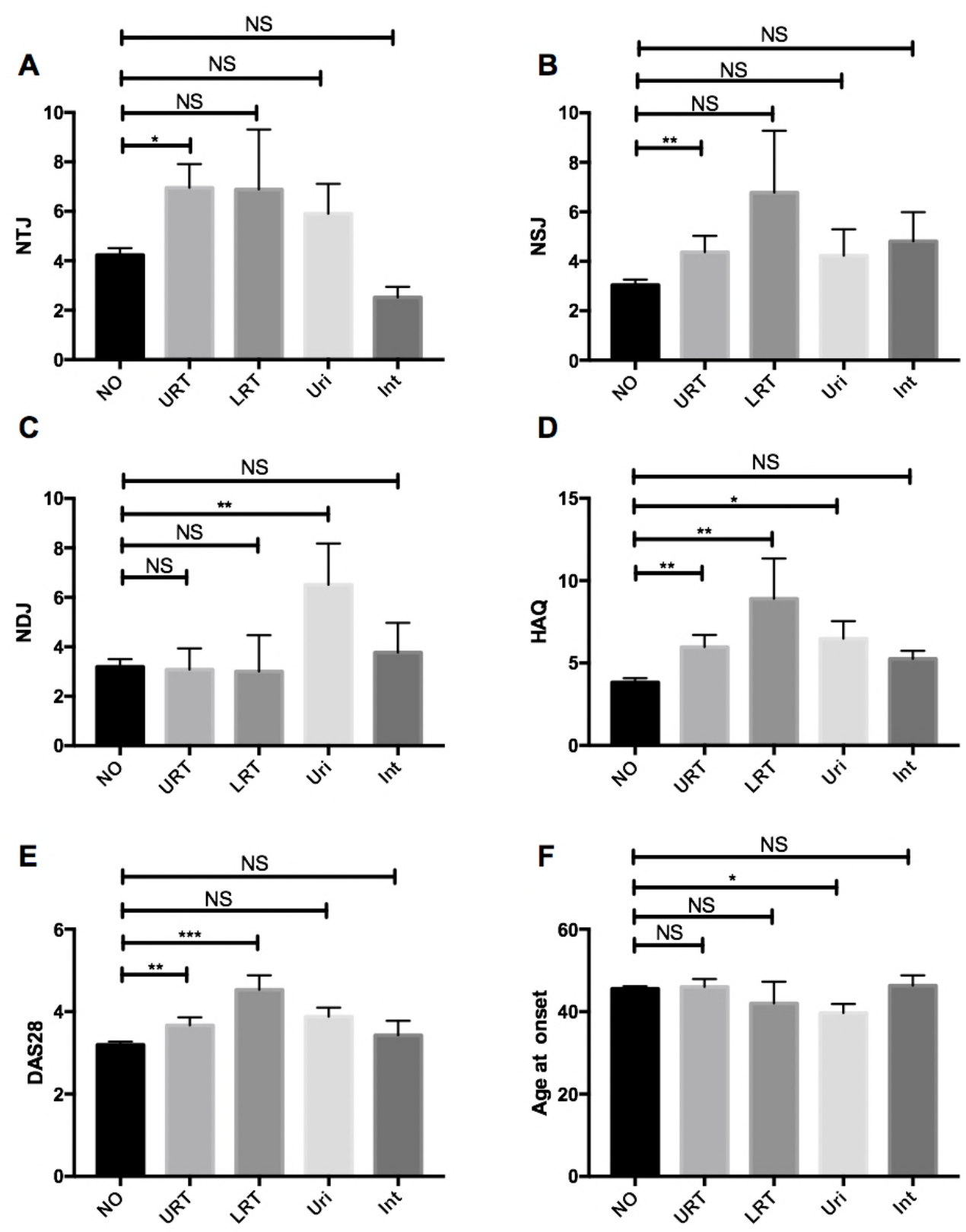

Fig1. Associations of disease activity and sites of infection. These patients were categorical into subgroups including no infection $(n=568)$, upper respiratory tract infection $(n=120)$, low respiratory tract infection $(n=25)$, urinary infection $(n=89)$ or intestinal infection $(n=100)$. Comparisons between groups were performed using the t-test or nonparametric test. The numbers of tender (A) and swollen (B) joints, HAQ (D) and DAS28 (E) were higher among respiratory tract infection, and number of deformity joints (C) was higher in urinary infection group. (F) Age at onset was younger in urinary infection group than other infection groups. (NO, no infection; URT, upper respiratory tract; LRT, lower respiratory tract; Uri, Urinary; Int, Intestinal) $(*: \mathrm{P}<0.05 ; * *: \mathrm{P}<0.01 ; * * *: \mathrm{P}<0.001)$ 

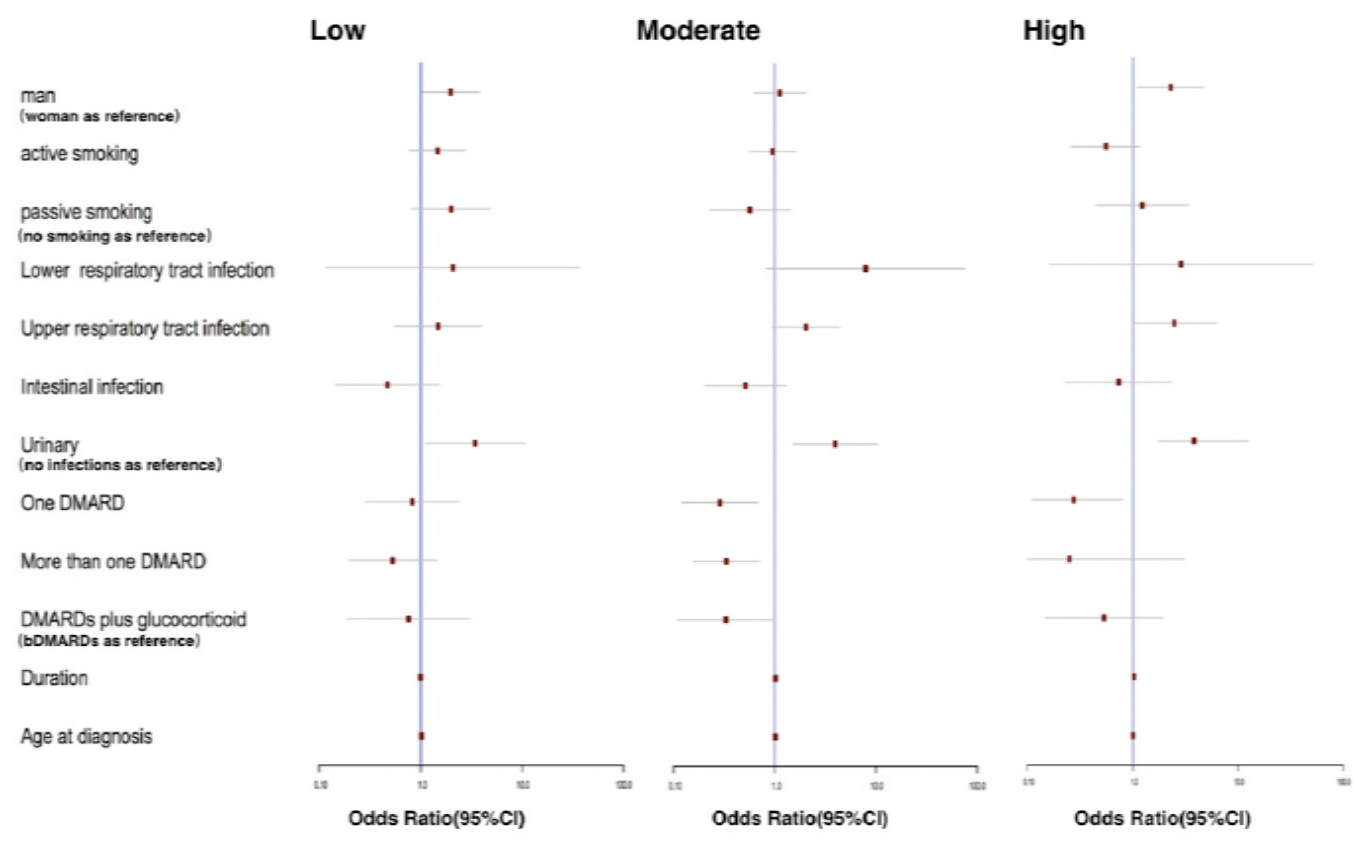

Error bars indicate $95 \%$ confidence intervals.

Figure2. Multinomial Logistic regression for the potential risk factors for high disease activity. (Remission as reference) 